\title{
Expression levels of IL-15 and IL-17 in synovial fluid of rheumatoid arthritis animal model
}

\author{
YI JIN, XIAO CHEN, ZONGYAN GAO, KE LIU, YI HOU and JIA ZHENG \\ Department of Orthopedics, People's Hospital of Zhengzhou University, \\ Henan Provincial People's Hospital, Zhengzhou, Henan 450003, P.R. China
}

Received January 1, 2018; Accepted July 25, 2018

DOI: 10.3892/etm.2018.6643

\begin{abstract}
The aim of the present study was to investigate the expression levels of interleukin-15 (IL-15) and interleukin-17 (IL-17) in synovial fluid of rheumatoid arthritis (RA) animal model, and to investigate their correlations with RA. A total of 100 Wistar rats were selected, among which 60 rats were used to establish the collagen II-induced arthritis (CIA) model as the model observation group, and the remaining 40 rats were used as blank control group. The levels of IL-15 and IL-17 in synovial fluid were detected via enzyme-linked immunosorbent assay (ELISA) at 1, 7, 14, 21 and 28 days after successful modeling. RA was evaluated by using arthritis index (AI) and pedal swelling volume. The expression levels of IL-15 and IL-17 in synovial fluid of rats in model observation group were higher than those in blank control group $(\mathrm{P}<0.05)$, and the levels of IL-15 and IL-17 in model observation group were gradually increased over time. In model observation group at 7 days after modeling, AI and pedal swelling volume began to be increased gradually reaching a peak at 28 days. The pedal swelling volume of CIA model rats was significantly higher than that of the blank control group $(\mathrm{P}<0.05)$. The increased expression levels of IL-15 and IL-17 in synovial fluid of rats in the CIA model observation group are correlated with the activity of disease, which can be used as reference indexes for the activity of RA.
\end{abstract}

\section{Introduction}

Rheumatoid arthritis (RA) is a kind of chronic connective tissue immune disease characterized by joint lesions, and it is also a kind of progressive arthritis. When RA occurs in the human body, it often causes joint pain and discomfort, joint space narrowing, and joint synovial swelling and pain, accompanied

Correspondence to: Dr Jia Zheng, Department of Orthopedics, People's Hospital of Zhengzhou University, Henan Provincial People's Hospital, 7 Weiwu Road, Zhengzhou, Henan 450003, P.R. China E-mail: jzc7k5@163.com; zhengjia90180@sina.com

Key words: rheumatoid arthritis, arthritis index, pedal swelling volume, IL-15, IL-17 with synovial effusion. In the early stage of disease, patients often do not pay attention to it, thus the treatment is delayed; as the disease continues to be aggravated, it will even cause disability. The prevalence rate of RA is approximately $0.5-1 \%$ in the world, and its incidence ratio in men and women is approximately 1:3. Moreover, RA can occur in any age group, and occurs frequently in people aged 30-50 years old (1). According to the research of Fessler et al (2), expression levels of inflammatory cytokine in human body are increased under the activity of RA.

Interleukin-15 (IL-15) and interleukin-17 (IL-17) are currently considered as cytokines for inflammatory response, which initiates inflammatory responses involving a series of downstream molecules, such as IL-6, IL-8 and granulocytemacrophage colony-stimulating factor (GM-CSF), and triggers inflammation (3). IL-15, an inflammatory cytokine existing in human cells and tissues, is produced by macrophages, and with similar functions and biological activities to IL-2, its role in immune diseases and inflammatory responses in human body has attracted increasingly more attention (4,5). IL-17 is a cytokine during the process that $\mathrm{T}$ lymphocytes induce and promote the inflammation, which has the functions of promoting immune response, inflammation development and hematopoiesis (6). Moreover, IL-17 can promote the production of reactive protein and plays an important regulatory role in the occurrence and development of inflammation (7); it can also induce other inflammatory cytokines, adhesion molecules, to play its roles in the occurrence and development of RA (8). It thus appears that IL-15 and IL-17 may be involved in the development process of RA.

The aim of the study was to examine the expression levels of IL-15 and IL-17 in synovial fluid of collagen II-induced arthritis (CIA) model rats, and explore their changes in RA through establishing CIA rat model, so as to provide clinical references for the diagnosis and treatment of RA.

\section{Materials and methods}

Experimental animals. A total of 100 healthy and clean Wistar rats aged approximately 6 weeks and weighing approximately $180 \mathrm{~g}$ were purchased from Shanghai Slac Laboratory Animal Co., Ltd., Shanghai, China (laboratory animal license no.: SDLB 2013-006). The rats were housed in a temperature controlled room $\left(21 \pm 2^{\circ} \mathrm{C}\right)$ on a 12:12-h light/dark cycle (lights 
Table I. General data of rats in the model observation and blank control groups.

\begin{tabular}{lccrr}
\hline Item & Model observation group & Blank control group & $\mathrm{t} / \chi^{2}$ & P-value \\
\hline Male $(\%)$ & $23(0.43)$ & $18(0.45)$ & $\chi^{2}=5.16$ & 0.349 \\
Female (\%) & $30(0.57)$ & $22(0.55)$ & $\chi^{2}=5.20$ & 0.351 \\
Age (days) & $39.57 \pm 2.61$ & $40.07 \pm 2.25$ & $\mathrm{t}=2.15$ & 0.186 \\
Weight (g) & $176.16 \pm 20.32$ & $180.26 \pm 16.37$ & $\mathrm{t}=4.06$ & 0.273 \\
Indoor temperature $\left({ }^{\circ} \mathrm{C}\right)$ & $23.12 \pm 0.63$ & $23.08 \pm 0.56$ & $\chi^{2}=1.97$ & 0.156 \\
Indoor humidity $(\%)$ & $52.45 \pm 5.03$ & $51.45 \pm 6.13$ & $\chi^{2}=2.06$ & 0.175 \\
\hline
\end{tabular}

on at 06:00). All rats had free access to water and food. This experimental study strictly adhered to the welfare ethical principle of animal experiments. This study was approved by the Animal Ethics Committee of Zhengzhou University Animal Center (Zhengzhou, China).

Main experimental reagents and instruments. Natural bovine type II collagen (containing acetic acid) was purchased from Beijing Biolead Biotechnology Co., Ltd., Beijing, China; Freund's complete adjuvant was purchased from Shanghai Qcbio Science and Technologies Co., Ltd., Shanghai, China. The IL-15 and IL-17 enzyme-linked immunosorbent assay (ELISA) kit was purchased from Shanghai Kanglang Biological Technology Co., Ltd., Shanghai, China; microplate reader was purchased from Shanghai Kanglang Biological Technology Co., Ltd.; GT10-1 high-speed centrifuge was purchased from Changsha Xiangzhi Instrument Co., Ltd., Changsha, China; Olympus CX23 microscope was purchased from Beijing Cossim Scientific Instruments Co., Ltd., Beijing, China; YLS-7C pedal volume measuring instrument was purchased from Chengdu Techman Software Co., Ltd., Chengdu, China.

Preparation of animal model. In this experiment, rats were modeled. A total of $100 \mathrm{Wistar}$ rats were divided into model observation group $(n=60)$ and blank control group $(n=40)$. CIA model was established in model observation group following the modeling method below: $20 \mathrm{mg}$ bovine type II collagen (containing acetic acid) was taken and added into $0.2 \mathrm{~mol} \cdot \mathrm{l}^{-1}$ acetic acid solution; the mixture was mixed evenly to be prepared into $4 \mathrm{mg} \cdot \mathrm{ml}^{-1}$ collagen solution, and placed in a refrigerator at $4-6^{\circ} \mathrm{C}$ overnight. Then $4 \mathrm{mg} \cdot \mathrm{ml}^{-1}$ collagen solution and Freund's complete adjuvant were fully fused in the ice bath and prepared into $2 \mathrm{~mol} \cdot \mathrm{l}^{-1}$ collagen emulsion; the emulsion was placed in a refrigerator at $4-6^{\circ} \mathrm{C}$ for standby application. Subsequently, $1.2 \mathrm{ml}$ collagen emulsion was extracted by using a needle tube, and subcutaneously injected into 60 rats via multiple points in tail root. After 1 week, $0.6 \mathrm{ml}$ collagen emulsion was injected to enhance the immunity. The 40 rats in the blank group did not receive any treatment in this experiment.

Detection of IL-15 and IL-17 in synovial fluid. In this experimental study, IL-15 and IL-17 levels in synovial fluid of rats were detected via ELISA. At 6:00 to 8:00 in the morning, $2 \mathrm{ml}$ synovial fluid was extracted from the joint cavity of rats into a test tube by using a syringe, and centrifuged at 2,200 x $\mathrm{g}$ for $15 \mathrm{~min}$. The supernatant was taken and stored in a refrigerator at $-80^{\circ} \mathrm{C}$. This experiment was performed in strict accordance with instructions of kits. Standard solution (100 $\mu \mathrm{l})$, sample to be tested and negative/positive control solution were taken into the reaction plate by using a pipette, and then $100 \mu \mathrm{l}$ bioreaction antibody solution was quickly added. The plate was covered with membrane, and the mixture was mixed evenly and let stand for $40 \mathrm{~min}$. Each reaction well was added with $100 \mu 1$ streptavidin; the mixture was mixed evenly and let stand for $40 \mathrm{~min}$; the liquid in the reaction well was poured away, and cleaning solution was added into each reaction well, mixed evenly and shaken slowly for $1 \mathrm{~min}$. The liquid in the reaction well was poured away; the operation was repeated 5 times; $100 \mu \mathrm{l}$ reaction liquid $\mathrm{A}$ and $100 \mu \mathrm{l}$ reaction liquid $\mathrm{B}$ were added into each reaction well, and the mixture was mixed evenly and let stand in the dark for $5 \mathrm{~min}$. Then $100 \mu \mathrm{l}$ stop solution was quickly added into the reaction wells, followed by immediate detection. The optical density value of each well was detected by a microplate reader (Bio-Rad, Hercules, CA, USA) at a wavelength of $550 \mathrm{~nm}$. Detection methods of IL-15 and IL-17 were same.

Arthritis index (AI) evaluation. In this experiment, AI integral method (9) was used for evaluation, and it was evaluated at $1,7,14,21$ and 28 days after rat modeling. According to the range of joint swelling and severity of joint deformity, AI was scored: no arthritis or no pedal swelling and deformity on the surface: 0 points; involvement of 1-2 plantar joints: $1-2$ points; involvement of 3-4 plantar joints: 3-4 points; involvement of more than 4 plantar joints: 5 points; severe arthritis: 5 points. There were a total of 20 points for four paws of each rat; the higher the AI score was, the severer the arthritis would be.

Detection of pedal swelling volume of rats. The anterior and posterior pedal swelling volumes of rats in model observation group and blank control group were measured at 1 day after modeling, and then the anterior and posterior pedal swelling volumes of rats were measured at 1, 7, 14, 21 and 28 days after modeling, respectively. The measurement method is as follows: Rats to be tested were placed in a fixed barrel, and the pedal swelling of rats were marked and scribed. The pedal volume of rats was measured using the YLS-7C pedal volume measuring instrument in strict accordance with the manufacturers' protocol. The larger the pedal swelling volume of rat was, the more serious the disease would be. 
Table II. Comparisons of changes in pedal swelling volumes of rats in model observation group and blank control group (mean \pm SD).

\begin{tabular}{|c|c|c|c|c|c|c|}
\hline Group & Qty (n) & 1 day & 7 days & 14 days & 21 days & 28 days \\
\hline Model observation group (ml) & 53 & $1.47 \pm 0.11$ & $1.53 \pm 0.13^{\mathrm{a}}$ & $1.58 \pm 0.16^{\mathrm{b}}$ & $1.60 \pm 0.13^{\mathrm{c}}$ & $1.63 \pm 0.20^{\mathrm{d}}$ \\
\hline Blank control group (ml) & 40 & $1.45 \pm 0.08$ & $1.46 \pm 0.11$ & $1.46 \pm 0.13$ & $1.47 \pm 0.11$ & $1.48 \pm 0.09$ \\
\hline $\mathrm{t}$ value & & 1.76 & 2.65 & 2.73 & 3.01 & 3.27 \\
\hline P-value & & 0.036 & 0.029 & 0.025 & 0.021 & 0.018 \\
\hline
\end{tabular}

Comparisons of pedal swelling volumes at 1, 7, 14,21 and 28 days after modeling in model observation group: in the comparison of pedal swelling volumes at 7 days and 1 day after modeling, ${ }^{a}=2.37, \mathrm{P}<0.05$; in the comparison of pedal swelling volumes at 14 and 7 days after modeling, ${ }^{\mathrm{b}} \mathrm{t}=2.46, \mathrm{P}<0.05$; in the comparison of pedal swelling volumes at 21 and 14 days after modeling, ${ }^{\mathrm{t}} \mathrm{t}=1.93$, $\mathrm{P}<0.05$; in the comparison of pedal swelling volumes at 28 and 21 days after modeling, ${ }^{\mathrm{d}}=2.09, \mathrm{P}<0.05$.

Table III. Comparisons of IL-15 levels in synovial fluid of rats in model observation group and blank control group (mean \pm SD).

\begin{tabular}{|c|c|c|c|c|c|c|}
\hline \multirow[b]{2}{*}{ Group } & \multirow[b]{2}{*}{ Qty (n) } & \multicolumn{5}{|c|}{ IL-15 (ng· $\left.\mathrm{ml}^{-1}\right)$} \\
\hline & & 1 day & 7 days & 14 days & 21 days & 28 days \\
\hline Model observation group & 53 & $21.61 \pm 5.37$ & $38.22 \pm 5.49^{\mathrm{a}}$ & $46.53 \pm 8.24^{\mathrm{b}}$ & $58.74 \pm 7.69^{c}$ & $73.26 \pm 9.13^{\mathrm{d}}$ \\
\hline Blank control group & 40 & $10.37 \pm 2.46$ & $13.37 \pm 4.02$ & $14.37 \pm 3.67$ & $13.37 \pm 4.79$ & $15.37 \pm 5.38$ \\
\hline t value & & 2.37 & 3.26 & 3.97 & 5.33 & 6.79 \\
\hline P-value & & 0.043 & 0.036 & 0.031 & 0.024 & 0.016 \\
\hline
\end{tabular}

Comparisons of IL-15 levels at 1, 7, 14, 21 and 28 days after modeling in model observation group: in the comparison of IL-15 levels at 7 days and 1 day after modeling, ${ }^{\mathrm{a}} \mathrm{t}=2.83, \mathrm{P}<0.05$; in the comparison of IL-15 levels at 14 and 7 days after modeling, ${ }^{\mathrm{b}} \mathrm{t}=3.13, \mathrm{P}<0.05$; in the comparison of IL-15 levels at 21 and 14 days after modeling, ${ }^{\mathrm{c}} \mathrm{t}=3.01, \mathrm{P}<0.05$; in the comparison of IL-15 levels at 28 and 21 days after modeling, ${ }^{\mathrm{d}} \mathrm{t}=2.93, \mathrm{P}<0.05$.

Statistical analysis. Statistical Product and Service Solutions (SPSS) 22.0 software (Beijing Strong-Vinda Information Technology Co., Ltd., Beijing, China) was used for analysis. Measurement data were presented as mean \pm standard deviation (mean $\pm \mathrm{SD}$ ). The t-test was used for the comparison of measurement data between two groups, Chi-square test was used for the comparison of rate. Logistics regression analysis was used for the correlation analysis. $\mathrm{P}<0.05$ was considered to indicate a statistically significant difference.

\section{Results}

Changes in general conditions and pedal swelling volumes of rats in two groups. In this experiment, after multi-point injection in tail roots of rats in model observation group, 53 out of 60 rats in model observation group were successfully modeled with the success rate of modeling of $88.33 \%$ (53/60). The sex and week age of rats, indoor temperature and indoor humidity had no effects on this experiment (P>0.05) (Table I). At 7 days after successful modeling, 53 rats in model observation group had different degrees of joint swelling, the hair began to become yellow slightly, the body weight was gradually decreased, the exercise capacity was also decreased, and they did not like to move. At 1, 7, 14, 21 and 28 days after modeling, the pedal swelling volumes of rats were $1.47 \pm 0.11,1.53 \pm 0.13,1.58 \pm 0.16$, $1.60 \pm 0.13$ and $1.63 \pm 0.20 \mathrm{ml}$, respectively. The pedal swelling volume of rats at 7 days after modeling was larger than that at 1 day after modeling $(\mathrm{P}<0.05)$. The pedal swelling volume at 14 days after modeling was larger than that at 7 days after modeling $(\mathrm{P}<0.05)$ and the pedal swelling volume at 21 days after modeling was larger than that at 14 days after modeling $(\mathrm{P}<0.05)$. The pedal swelling volume at 28 days after modeling was larger than that at 21 days after modeling $(\mathrm{P}<0.05)$. With the increase of time, the joint swelling volume was gradually aggravated until reaching the peak at 28 days. The pedal swelling volumes of rats in model observation group at 1,7 , 14, 21 and 28 days were significantly larger than those in blank control group $(\mathrm{P}<0.05)$ (Table II).

Comparisons of IL-15 and IL-17 in synovial fluid between two groups. Results of ELISA showed that the levels of IL-15 in model observation group at 1, 7, 14, 21 and 28 days after modeling were increased gradually, and the level was higher at 7 days after modeling than that at 1 day after modeling $(\mathrm{P}<0.05)$. The level of IL-15 at 14 days after modeling was higher than that at 7 days after modeling $(\mathrm{P}<0.05)$, and the level of IL-15 at 21 days after modeling was higher than that at 14 days after modeling $(\mathrm{P}<0.05)$. The level of IL-15 at 28 days after modeling was higher than that at 21 days after modeling $(\mathrm{P}<0.05)$. The concentrations of IL-15 in synovial fluid of rats in model observation group were higher than those in blank control group $(\mathrm{P}<0.05)$. The levels of IL-17 in model observation group at $1,7,14,21$ and 28 days after modeling were increased gradually, and the level of IL-17 was higher at 7 days after modeling 
Table IV. Comparisons of IL-17 levels in synovial fluid of rats in model observation group and blank control group (mean \pm SD).

\begin{tabular}{|c|c|c|c|c|c|c|}
\hline \multirow[b]{2}{*}{ Group } & \multirow[b]{2}{*}{ Qty (n) } & \multicolumn{5}{|c|}{$\mathrm{IL}-17\left(\mathrm{ng} \cdot \mathrm{ml}^{-1}\right)$} \\
\hline & & 1 day & 7 days & 14 days & 21 days & 28 days \\
\hline Model observation group & 53 & $18.92 \pm 3.51$ & $43.85 \pm 11.93^{\mathrm{a}}$ & $53.27 \pm 10.33^{\mathrm{b}}$ & $72.05 \pm 11.38^{c}$ & $86.46 \pm 10.28^{\mathrm{d}}$ \\
\hline Blank control group & 40 & $11.28 \pm 3.06$ & $11.37 \pm 4.28$ & $13.13 \pm 3.09$ & $14.37 \pm 3.23$ & $11.75 \pm 5.07$ \\
\hline t value & & 4.63 & 5.97 & 6.22 & 7.03 & 8.16 \\
\hline P-value & & 0.046 & 0.031 & 0.028 & 0.017 & 0.011 \\
\hline
\end{tabular}

Comparisons of IL-17 levels at 1, 7, 14, 21 and 28 days after modeling in model observation group: in the comparison of IL-17 levels at 7 days and 1 day after modeling, ${ }^{a} t=5.36, P<0.05$; in the comparison of IL-17 levels at 14 and 7 days after modeling, ${ }^{b} t=3.19, P<0.05$; in the comparison of IL-17 levels at 21 and 14 days after modeling, ${ }^{c}=4.26, P<0.05$; in the comparison of IL-17 levels at 28 and 21 days after modeling, ${ }^{\mathrm{d}} \mathrm{t}=3.76, \mathrm{P}<0.05$.

Table V. Comparisons of AI scores of rats between model observation group and blank control group (mean \pm SD).

\begin{tabular}{lccccc}
\hline Group & Qty (n) & 1 day & 7 days & 14 days & 21 days \\
\hline Model observation group & 53 & $3.27 \pm 0.65$ & $5.28 \pm 2.29$ & $8.26 \pm 2.36$ & $10.33 \pm 3.94$ \\
Blank control group & 40 & 0 & 0 & 0 & $13.63 \pm 4.77$ \\
P-value & & 0.032 & 0.029 & 0.024 & 0 \\
\hline
\end{tabular}

than that at 1 day after modeling $(\mathrm{P}<0.05)$; the level of IL-17 at 14 days after modeling was higher than that at 7 days after modeling $(\mathrm{P}<0.05)$; the level of IL-17 at 21 days after modeling was higher than that at 14 days after modeling $(\mathrm{P}<0.05)$; the level of IL-17 at 28 days after modeling was higher than that at 21 days after modeling $(\mathrm{P}<0.05)$. The concentrations of IL-17 in synovial fluid of rats in model observation group were higher than those in blank control group $(\mathrm{P}<0.05)$ (Tables III and IV).

Comparisons of AI scores between two groups. Rats in the model observation group began to have different degrees of joint swelling at 7-14 days after modeling. The swelling degree reached the peak after 4 weeks, and some joints were deformed (Table V).

Correlations of IL-15 and IL-17 expression levels in synovial fluid with pedal swelling volume and AI score of rats in model observation group. Correlations of IL-15 and IL-17 expression levels in synovial fluid with pedal swelling volume and AI score of rats in the model observation group were analyzed. It was found that the levels of IL-15 and IL-17 in synovial fluid were positively correlated with the pedal swelling volume and AI score of rats in the model observation group (Table VI).

\section{Discussion}

Rheumatoid arthritis (RA) is a kind of chronic connective tissue immune disease characterized by joint lesions. Its clinical symptoms are manifested as persistent swelling and pain in joints, often involving the interphalangeal joint, elbow joint and pedal joint (10). The joint swelling and pain in RA patients are severer in the morning, and they are slightly relieved at
Table VI. Correlations of IL-15 and IL-17 expression levels in synovial fluid with pedal swelling volume and AI score in model observation group.

\begin{tabular}{lcc}
\hline Items & IL-15 level & IL-17 level \\
\hline AI score & $0.357^{\mathrm{a}}$ & $0.236^{\mathrm{a}}$ \\
Pedal swelling volume & $0.274^{\mathrm{a}}$ & $0.356^{\mathrm{a}}$ \\
\hline
\end{tabular}

${ }^{\mathrm{a}} \mathrm{P}<0.05$.

noon and afternoon. Currently, the common predisposing factors in clinic are chronic infection, estrogen changes, humid and cold environment. RA is a systemic immune disease induced by multiple factors, and its incidence rate is not related to the region (11). At present, some data have indicated that a large number of inflammatory cytokines are involved in the development of RA, and the expression levels of IL-15 and IL-17 are significantly increased in RA patients (12).

In this study, it can be seen that the pedal swelling volumes and AI scores of rats at 1, 7, 14, 21 and 28 days showed increasing trends with the increase of time, indicating that $\mathrm{RA}$ is more and more serious during this period, and IL-15 and IL-17 levels are gradually increased. Therefore, the two inflammatory cytokines, IL-15 and IL-17, are involved in the development process of RA.

IL-15 plays a regulatory role in the activation of neutrophils, and can stimulate the production of cytokines, such as IL-1, IL-6 and monocyte chemoattractant protein (MCP), and promote the adhesion of leukocyte vascular endothelial cells and the accumulation of leukocytes in inflammation, thus 
expanding the inflammatory response; so it has various effects in inflammatory response and human immunity $(13,14)$. In this study, results showed that the expression levels of IL-15 in synovial fluid in the model observation group at 1, 7, 14, 21 and 28 days were gradually increased, and the levels of IL-15 in model observation group were significantly higher than those in the blank control group $(\mathrm{P}<0.05)$. Additionally, the IL-15 expression level was positively correlated with AI score and pedal swelling volume. Therefore, it can be concluded that there is a close relationship between IL-15 and the occurrence and development of RA, and IL-15 can be used as a judgment index for the development of RA. The possible reason is that IL-15 can promote T lymphocytes, B lymphocytes and natural killer cells for immune regulation, proliferation and differentiation, and promote and synthesize the human immunoglobulin, thus playing a lot of roles in the occurrence process of autoimmune diseases (15). Experiments of Zhang et al (16) also confirmed the view that the IL-15 level in synovial fluid is increased in RA patients, and IL-15 can be used as one of the judgment indexes for the activity of RA.

IL-17 is a cytokine during the process that $\mathrm{T}$ lymphocytes promote and induce the inflammation, and it has functions of promoting immune response, inflammation development and hematopoiesis (17). Results of the current study showed that the levels of IL-17 in model observation group at 1, 7, 14, 21 and 28 days were significantly higher than those in blank control group $(\mathrm{P}<0.05)$, and the level was positively correlated with AI score and pedal swelling volume. Therefore, it is concluded that IL-17 can be used as a judgment index for the development of RA. The possible reason is that IL-17 can release and promote inflammatory cytokines to expand the inflammatory response, enhance the absorption and synthesis of osteoclasts, and control the cartilage cells to secrete cartilage matrix, thereby resulting in bone erosion at the affected joints (18). In addition, IL-17 is closely associated and interacts with other inflammatory cytokines, eventually leading to the occurrence and development of RA in patients (4). According to the research of Al-Saadany et al (19), the expression levels of IL-15 and IL-17 in synovial fluid of RA patients are high, and there is a close relationship between IL-15 and IL-17; both of them can serve as judgment indexes for the activity of RA. It is also reported in the literature that IL-17 cannot be used as a judgment index for the activity of RA. For example, Al-Saadany et al (19) thought that IL-15 can be used as a judgment index for the activity of RA, but IL-17 is not associated with the activity of RA. The possible reason is that the differences in detection samples, instruments, reagents, and reference standards, affect the results.

In this experimental study, the repeatability and reliability of animal experiments were considered. To ensure the accuracy of results, the rats purchased were strictly screened, and the differences in sex, weight and health status, of Wistar rats were strictly controlled. In this experiment, sex and week age of rats, indoor temperature and indoor humidity had no effects on this experiment. Rats have light weight and small size, and they can be fed, managed and observed easily; moreover, rats have a good sensitivity on bacteria, viruses, external stimuli and inflammatory response, and changes in morphology, hair, exercise capacity and pedal swelling volume of rats can be carefully observed in the development of RA; therefore, rats were selected as the modeling objects in this study. Rats are still different from human body in the tissue structure, physiological characteristics, system function and disease characteristics. In medical experimental research, animal experiments serve for human beings, and the results of animal experiments should be integrated into the human body. Animals and humans are not the same genus after all; consequently, effective results in animals are not necessarily effective in human, while the ineffective results in animals are not necessarily ineffective in human body. Therefore, it is hoped that clinical patients with RA can be selected as subjects for experiments in the future to further prove the results of the present study.

In conclusion, the results have shown that the increased levels of IL-15 and IL-17 in synovial fluid of Wistar rats in CIA model observation group were associated with the development of RA. IL-15 and IL-17 expression levels can be used as judgment indexes for the activity of RA clinically.

\section{Acknowledgements}

Not applicable.

\section{Funding}

This study was supported by Henan Province Science and Technology Breakthrough Plan (project no.: 162102310300; project name: MRI evaluation of postoperative complications after artificial hip and knee arthroplasty).

\section{Availability of data and materials}

All data generated or analyzed during this study are included in this published article.

\section{Authors' contributions}

YJ and XC designed the study. ZG and KL established the animal models. YH and JZ performed ELISA assay. YJ prepared the manuscript. All authors read and approved the final manuscript.

\section{Ethics approval and consent to participate}

This study was approved by the Animal Ethics Committee of Zhengzhou University Animal Center (Zhengzhou, China).

\section{Patient consent for publication}

Not applicable.

\section{Competing interests}

The authors declare no competing interests.

\section{References}

1. Massalska M, Plebanczyk M, Radzikowska A, Kucawarnawin E, Prochorecsobieszek M, Musialowicz U, Skalska U, Kurowska W, Kornatka A and Burakowski T: A2.06 Functional regulatory T-cells in rheumatoid arthritis bone marrow are modulated by IL-15 and strong antigenic stimulation. Ann Rheum Dis 75: A17.12-A17, 2016. 
2. Fessler J, Raicht A, Husic R, Ficjan A, Schwarz C, Duftner C, Schwinger W, Graninger WB, Stradner MH and Dejaco C: Novel senescent regulatory T-cell subset with impaired suppressive function in rheumatoid arthritis. Front Immunol 8: 300, 2017.

3. Saito S, Suzuki K, Yamaoka K, Amano K, Tokuhira M and Takeuchi T: FRI0168 restoration of decreased lymphocytes, $\mathrm{CD}^{+} \mathrm{T}$ cell subsets with $\mathrm{TH} 1$ skewed phenotype associate with spontaneous regression of lympho-proliferative disorders in rheumatoid arthritis patients treated with methotrexate Ann Rheum Dis 76 (Suppl 2): 544, 2017. http://ard.bmj.com/ content/76/Suppl_2/544.2.

4. Ndongo-Thiam $\bar{N}$, Clement A, Pin JJ, Razanajaona-Doll D and Miossec P: Negative association between autoantibodies against IL-17, IL-17/anti-IL-17 antibody immune complexes and destruction in rheumatoid arthritis. Ann Rheum Dis 75: 1420-1422, 2016.

5. Bendele A: Animal models of rheumatoid arthritis. J Musculoskelet Neuronal Interact 1: 377-385, 2001.

6. Smolen JS, Schöls M, Braun J, Dougados M, FitzGerald O, Gladman DD, Kavanaugh A, Landewé R, Mease P, Sieper J, et al Treating axial spondyloarthritis and peripheral spondyloarthritis, especially psoriatic arthritis, to target: 2017 update of recommendations by an international task force. Ann Rheum Dis 77 3-17, 2018.

7. Castrejon I, Chua J, Malfait AM, Block JA and Pincus T: FRI0081 comparison of disease burden in patients with rheumatoid arthritis (RA), RA with secondary osteoarthritis (OA), and OA in routine care. Ann Rheum Dis 75 (Suppl 2): 456, 2016. http://ard.bmj.com/content/75/Suppl_2/456.2.

8. Younes SA, Freeman ML, Mudd JC, Shive CL, Reynaldi A, Panigrahi S, Estes JD, Deleage C, Lucero C, Anderson J, et al: IL-15 promotes activation and expansion of $\mathrm{CD} 8^{+} \mathrm{T}$ cells in HIV-1 infection. J Clin Invest 126: 2745-2756, 2016.

9. Cappelleri JC, Bushmakin AG, Whipple S, Hlavacek P, Sadosky A and Willke RJ: Mapping the Western Ontario and Mcmaster Universities Arthritis Index (WOMAC) to the EQ-5D. Value Health 19: A235-A236, 2016.

10. Iacomino G, Marano A, Stillitano I, Aufiero VR, Iaquinto G, Schettino M, Masucci A, Troncone R, Auricchio S and Mazzarella G: Celiac disease: Role of intestinal compartments in the mucosal immune response. Mol Cell Biochem 411: 341-349, 2016.
11. Rosario M, Liu B, Kong L, Collins LI, Schneider SE, Chen X, Han K, Jeng EK, Rhode PR, Leong JW, et al: The IL-15-based ALT-803 complex enhances FcyRIIIa-triggered NK cell responses and in vivo clearance of B cell lymphomas. Clin Cancer Res 22: 596-608, 2016.

12. Siebert S, Tsoukas A, Robertson J and McInnes I: Cytokines as therapeutic targets in rheumatoid arthritis and other inflammatory diseases. Pharmacol Rev 67: 280-309, 2015.

13. Friday SC and Fox DA: Phospholipase D enzymes facilitate IL-17- and TNF $\alpha$-induced expression of proinflammatory genes in rheumatoid arthritis synovial fibroblasts (RASF). Immunol Lett 174: 9-18, 2016.

14. Meednu N, Zhang H, Owen T, Sun W, Wang V, Cistrone C, Rangel-Moreno J, Xing L and Anolik JH: Production of RANKL by memory B cells: A link between B cells and bone erosion in rheumatoid arthritis. Arthritis Rheumatol 68: 805-816, 2016.

15. Na HS, Kwon JE, Lee SH, Jhun J, Kim SM, Kim SY, Kim EK, Jung K, Park SH and Cho ML: Th17 and IL-17 cause acceleration of inflammation and fat loss by inducing $\alpha 2$-glycoprotein 1 (AZGP1) in rheumatoid arthritis with high-fat diet. Am J Pathol 187: 1049-1058, 2017.

16. Zhang Y, Li Y, Lv TT, Yin ZJ and Wang XB: Elevated circulating Th17 and follicular helper CD4(+) T cells in patients with rheumatoid arthritis. APMIS 123: 659-666, 2015.

17. Wedekind KJ, Ruff KJ, Atwell CA, Evans JL and Bendele AM: Beneficial effects of natural eggshell membrane (NEM) on multiple indices of arthritis in collagen-induced arthritic rats. Mod Rheumatol 27: 838-848, 2017.

18. Finckh A, Alpizar-Rodriguez D and Roux-Lombard P: Value of biomarkers in the prevention of rheumatoid arthritis. Clin Pharmacol Ther 102: 585-587, 2017.

19. Al-Saadany HM, Hussein MS, Gaber RA and Zaytoun HA: Th-17 cells and serum IL-17 in rheumatoid arthritis patients: Correlation with disease activity and severity. Egyp Rheumatol 38: 1-7, 2016.

This work is licensed under a Creative Commons

Attribution-NonCommercial-NoDerivatives 4.0 International (CC BY-NC-ND 4.0) License. 